\title{
La factura electrónica en Colombia: visión integral
}

Fecha de recepción: 3 de junio de 2011

Fecha de aprobación: 8 de julio de 2011

\author{
Jorge Mario Calvo \\ jmcalvo@ubiquando.com.co \\ Universidad de la Sabana
}

Ingeniero de Sistemas de la Universidad Distrital de Colombia, magíster en Ingeniería de Sistemas y especialista en Telemática de la Universidad de los Andes. Tiene un DEA en Infirmática: sistema y comunicación de la Universidad de Grenoble I en Francia. Es el gerente y fundador de Ubiquando Colombia (1993).

\section{Alex Vicente Chacón Jiménez}

alex.chacon@software-colombia.com

Universidad de la Sabana

Ingeniero de sistemas de la Universidad de los Andes y magíster en ingeniería de sistemas de la misma Universidad. Se especializó en construcción de software y aseguramiento criptográfico de aplicaciones en la Universidad de los Andes. Es director de tecnología Software de Colombia S.A.S. y consultor de tecnología de la Superintendencia Nacional de Salud. Además es profesor de Desarrollo de aplicaciones móviles y estructuras de datos en la Universidad de la Sabana. Fue diseñador e implementador de soluciones PKI y de firma digital en el Estado colombiano como consultor de tecnología de Certicámara S.A. del año 2005 al 2009.

\section{Resumen}

En el presente documento de análisis se da a conocer la factura electrónica en el contexto colombiano enfocado desde varios puntos de vista y evaluado desde distintas áreas del conocimiento. En primera medida se pretende dar una visión integral del concepto en el cual se abarquen consideraciones jurídicas, técnicas, económicas y su viabilidad de implementación en diferentes escenarios en la pequeña, mediana y gran industria. Los conceptos y observaciones mencionados en el presente artículo de investigación responden

\begin{abstract}
This analysis paper presents the electronic billing within the Colombian context from several points of view, and evaluated from different areas of knowledge. First, it is intended to give an integral vision of the concept in which legal, technical, and economic considerations are taken into account as well as their implementation viability in different scenarios on the small, medium, and big industry. The concepts and observations mentioned in this research paper answer a set of experiences in the design and implementation of an
\end{abstract}


a un conjunto de experiencias en el diseño e implementación de una herramienta de facturación electrónica que responde a un conjunto de necesidades de negocio y a las disposiciones legales definidas por el Estado colombiano.

\section{Palabras clave}

Factura electrónica, Colombia, DIAN, Decreto 1929 del 29 de Mayo de 2007, Ley de Comercio Electrónico 527 de 1999, PKI. electronic billing tool that responds to the business needs and legal dispositions defined by the Colombian Government.

\section{Keywords}

Electronic Bill, Colombia, DIAN of Electronic Law Commerce 


\section{Introducción}

El 29 de mayo del 2007 el Ministerio de Hacienda y Crédito Público emitió el decreto 1929 con el fin de darle a la industria colombiana una segunda oportunidad (esta vez una oportunidad de viable implementación y adopción, tanto para la pequeña, mediana y aún la gran industria) en la que se permite modernizar y optimizar los procesos de facturación y la relación tributaria con el Estado, adoptando nuevas tecnologías de información en medios totalmente electrónicos. Por su parte, la Dirección de Impuestos y Aduanas Nacionales (DIAN) emitió el 28 de Noviembre del 2007 la resolución 14465 en la cual se establecen las características y contenido técnico de las facturas electrónicas y de las notas crédito. De la misma manera, junto con la resolución 14465, emitió cinco anexos técnicos en los cuales se dan a conocer las directivas suficientes y necesarias para calcular un contenido técnico de control para cada una de las facturas electrónicas y reportar la actividad de facturación electrónica por parte del facturador al ente de control y fiscalización tributaria del Estado: la DIAN.

Como primer punto de análisis, tenemos el contenido técnico de control de las facturas electrónicas y las notas crédito. Su cálculo se define en el anexo técnico No. 1 de la resolución 14465 y su objetivo es "garantizar" la integridad del documento electrónico por medio de un algoritmo dado. En este algoritmo se concatenan los valores relevantes de la factura en una sola unidad de información. Para la DIAN, la información relevante de una factura comprende: (i) número de la factura, (ii) fecha de emisión, (iii) hora de emisión, (iv) valor de la factura, (v) valor del IVA, (vi) NIT del obligado a facturar (vii) tipo de documento del adquiriente, (viii) número de documento del adquiriente y (ix) clave de contenido técnico de control. Esta última clave la asigna la DIAN a cada uno de los obligados a facturar que opten por este medio.

Posteriormente, al resultado de esta concatenación de información relevante, se le calcula un resumen numérico por medio de un algoritmo denominado SHA1. El resultado de este cálculo es tratado como el contenido técnico de control de la factura y de las notas crédito y le sirve a la DIAN como asegurador de la integridad de la información contenida en una factura electrónica.

En los anexos técnicos 2, 4 y 5, la DIAN define de manera muy diligente el formato que se deberá respetar en los reportes bimensuales que un obligado a facturar deberá enviar a este organismo de vigilancia y control. En el anexo técnico No. 2 se encuentra definido un reporte consolidado por adquiriente. En este reporte la empresa emisora de facturación electrónica informa a la DIAN a quién y por cuánto se ha facturado. En el anexo técnico No. 4 se informan las novedades en los acuerdos de facturación. Una empresa emisora de facturación no podrá utilizar el modelo electrónico sin antes contar con el consentimiento del adquiriente. $\mathrm{Y}$ en el anexo técnico No. 5 se encuentra definido un informe detallado de facturas por parte del obligado a facturar, reporte que podrá exigir la DIAN a voluntad y que contiene información detallada de la actividad de facturación. Por su parte, el anexo técnico No. 3 sólo aplica para las personas a las cuales se les ha facturado en modalidad 
electrónica (adquirientes) y que quieren hacer valer ante la DIAN costos y deducciones. En este reporte el adquiriente informa a la DIAN quién y por cuánto le han facturado.

Ahora bien, si un sistema de información puede generar documentos electrónicos con un contenido de control técnico calculado a partir de las directivas dadas en el anexo técnico No. 1 de la resolución 14465 y adicionalmente permite generar los reportes de emisión de facturación tal como los solicita la DIAN en sus anexos técnicos 2 al 5, no se puede considerar que ya se ha implementado lo mínimo en el proceso de facturación electrónica. Existen requerimientos funcionales y no funcionales adicionales que deben responder a la normatividad vigente y a las necesidades de negocio en un contexto empresarial colombiano y aún latinoamericano. Por esta razón, en el presente documento de análisis se desglosarán aspectos de diseño e implementación enmarcados en una experiencia de construcción de una herramienta que responde a las necesidades tanto del sector empresarial en la pequeńa mediana y gran industria, como de los entes de control y vigilancia en el sector tributario del Estado colombiano.

\section{Antecedentes}

Existen tres hitos importantes en el proceso evolutivo de la factura electrónica en Colombia. La primera referencia se remonta al año de 1996 en el cual se reglamentó el uso de la factura electrónica como un documento computacional que soporta una transacción de venta de bienes o prestación de servicios.
Por otro lado, México y Chile responden a dos paradigmas estratégicos en la adopción de nuevas tecnologías en sus procesos mercantiles y tributarios que les permitió a las industrias de ambos países aumentar la competitividad, reduciendo los costos de facturación y los niveles de evasión de impuestos.

\section{A. Decreto 1094 de 1996}

Fue el primer intento del Gobierno colombiano en el uso de medios totalmente electrónicos para soportar las operaciones mercantiles de compraventa de productos y/o servicios.

Para esta primera versión de factura electrónica se definió el estándar EDIFACT como el lenguaje universal de transferencia e intercambio de información.

EDIFACT (Electronic Data Interchange For Administration, Commerce and Transport) representa un estándar desarrollado por las Naciones Unidas para el intercambio de documentos comerciales. Se encuentra estructurado jerárquicamente por una serie de segmentos de información que siguen reglas de sintaxis definidas. A su vez, estos segmentos se encuentran conformados por elementos de datos (simples o compuestos). Un elemento de datos compuesto se encuentra conformado, a su vez, por elementos de datos simples que pueden estar codificados o no codificados. Un elemento de datos codificado toma sus valores de una lista de códigos determinada $a^{[\mathrm{n}]}$. La jerarquía del estándar se denota a continuación: 


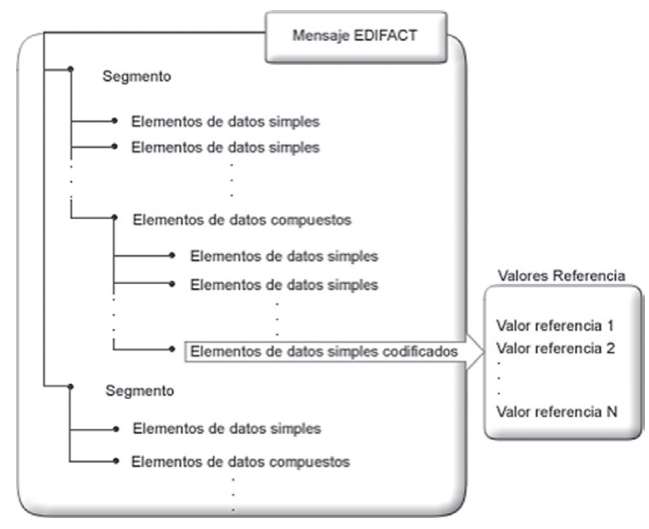

Sin embargo, no hay nada mejor que un buen ejemplo para entender, a grosso modo, cómo aplicar las reglas de construcción de mensajes EDIFACT en el intercambio de facturación electrónica. Nótese la estructura de la siguiente factura tradicional:

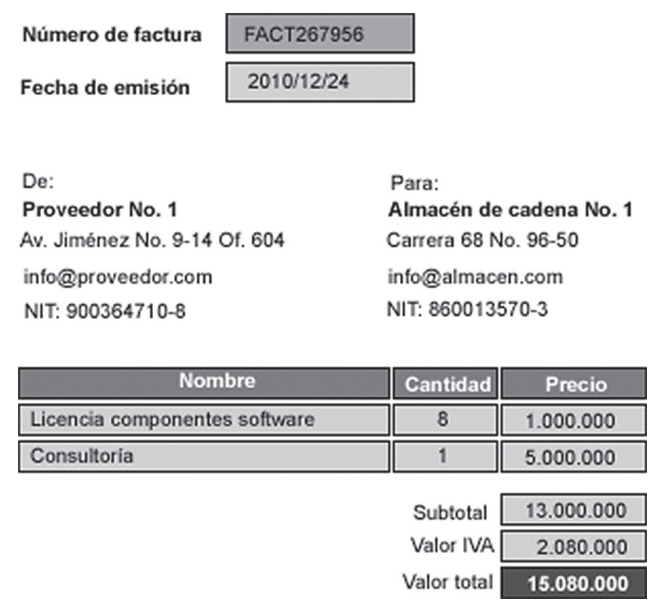

Esta factura se puede modelar con el estándar EDIFACT por medio de los siguientes segmentos y elementos de datos simples:
- UNH: cabecera del mensaje.

- Datos: número de factura y fecha de emisión.

- NAD: segmento de definición de empresa.

- Datos: razón social, dirección, email y NIT.

- ART: segmento de definición de artículo.

- Datos: nombre de artículo y cantidad.

- PRI: segmento de definición de precio.

- Datos: precio unitario del artículo precedente.

- TOT: importes monetarios.

- Datos: sub total de la factura y valor total del IVA.

- UNT: final del mensaje.

- Datos: número de segmentos del mensaje.

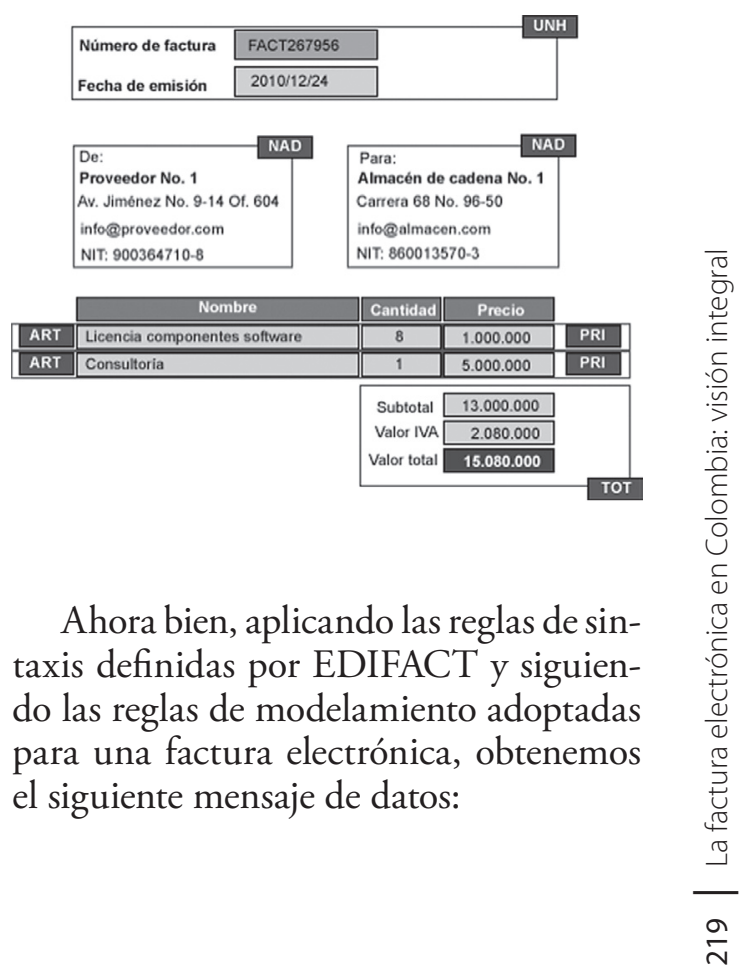


'UNH+FACT267956+20101224

'NAD+Proveedor No. 1+Av. Jiménez

No.9-14 Of.604+info@proveedor. com+900364710-8

'NAD+Almacén de cadena No.

1+Carrera 68 No.96-50+info@alma-

cen.com+860013570-3

'ART+Licencia componentes

software+8

'PRI+1.000.000

'ART+Consultoría+1

'PRI+5.000.000

'TOT+13.000.000+2.080.000+15.0

80.00

'UNT+9

Finalmente, en el artículo No. 3 del decreto 1094 de 1996 se definió el concepto de las Empresas administradoras de redes de valor agregado las cuales, previa autorización del Ministerio de Comunicaciones y registro ante la Subdirección de Fiscalización de la Dirección de Impuestos y Aduanas Nacionales (DIAN), podían intermediar en el intercambio de mensajes de datos electrónicos. Adicionalmente, estos intermediarios autorizados debían disponer ante la DIAN de un buzón electrónico fiscal en el cual existiría registro, custodia y organización de todos los mensajes electrónicos (facturas) para los cuales sirvieron como puente de comunicación.

\section{B. Legislación chilena}

El caso chileno es considerado un caso de éxito en América latina y es fuente de inspiración en la creación de las nuevas legislaciones en las cuales se quieren aprovechar los medios electrónicos emergentes, para simplificar y optimizar los procesos de facturación y fiscalización tributaria.
De acuerdo a la resolución exenta SII (Servicio de Impuestos Internos, equivalente de la DIAN en Chile) No. 45 del 1 de septiembre de 2003, en la cual se establecen las normas y procedimientos de operación respecto de los documentos tributarios electrónicos, el modelo de facturación electrónica chileno se describe de la siguiente manera:

\section{Enrolamiento}

Para operar en la modalidad de factura electrónica los contribuyentes deberán estar enrolados en el SII como emisores de documentos electrónicos. Esto no los obligará a generar todos sus documentos en forma electrónica pero sí a recibir documentos electrónicos de otros emisores. Los contribuyentes enrolados podrán conseguir la autorización de sus folios a través del Web del SII, y, utilizando esos folios podrán emitir, transmitir y almacenar sus documentos tributarios en forma electrónica.

\section{Generación}

Cada documento debe ser generado en el estándar definido por las especificaciones del SII. Debe incorporar una firma electrónica digital de la totalidad del documento, la que permitirá asegurar la identidad del emisor y cautelar la integridad del documento. Como resguardo adicional, se exigirá incorporar un timbre electrónico, el que se imprimirá en código de barras en la representación impresa de los documentos. Este timbre electrónico, obtenido según un algoritmo de 
seguridad especificado por el SII, permite a los fiscalizadores verificar fuera de linea, en los controles móviles, la validez de los documentos impresos que acompañan mercaderías.

\section{Envío}

El contribuyente deberá enviar el documento al SII, vía Internet, antes de que sea recibido por su destinatario o utilizado para el transporte físico de bienes. El contribuyente emisor debe enviar el documento al receptor, ya sea manual o electrónico. Al receptor manual, no enrolado en el sistema, le debe enviar la representación en papel del documento, la que este último sí está obligado a almacenar.

\section{Almacenamiento}

Los contribuyentes enrolados en el sistema requerirán almacenar los documentos tributarios electrónicos emitidos y recibidos sólo en forma electrónica y estarán eximidos de la obligación de almacenar dichos documentos en papel para una posible revisión del SII.

\section{Verificación}

El Servicio de Impuestos Internos habilitará una verificación de documentos en su sitio Web, lo que permitirá a los contribuyentes receptores y a los fiscalizadores del SII, cerciorarse de la validez de un documento.

\section{Legislación mexicana}

En México, la facturación electrónica se remonta al año 2004 de acuerdo a los lineamientos legales establecidos por el SAT (Servicios de Administración Tributaria) en el Anexo No. 20 de la Resolución Miscelánea Fiscal.

La legislación mexicana define la factura electrónica como un documento en formato XML que deberá presentar una firma digital para garantizar la integridad, autenticidad y no repudiación de contenidos, un resumen del contenido de la factura y un folio con el cual se indica el número de la transacción.

Posteriormente, el 28 de junio de 2006, se establecieron las bases para la prestación de los servicios de emisión y envío de facturación electrónica con la reforma del Código Fiscal de la Federación. En su legislación, el SAT anunció tres formas de facturar electrónicamente en México:

- Facturación por medios propios: consiste en la generación de facturación electrónica utilizando la infraestructura tecnológica (hardware/software) de la empresa emisora.

- Facturación por medio de un proveedor autorizado por el SAT: en donde la facturación electrónica es emitida, entregada y conservada por un tercero autorizado por el SAT.

- Facturación por medio de la aplicación gratuita del SAT- Micro-E: es un servicio sin costo creado para personas naturales o jurídicas (cuyos ingresos anuales no superen los cuatro millones de pesos mexicanos) que les permite generar facturación, llevar el control de operaciones y llevar el control de obligaciones fiscales. 
Finalmente y de acuerdo a las modificaciones del código fiscal mexicano, a partir del primero de enero del 2011 se hará obligatorio el uso de la facturación electrónica para todas las operaciones de ventas de productos y/o servicios superiores a 2 mil pesos mexicanos con lo cual se da un impulso definitivo por parte del Estado a la adopción de nuevas tecnologías en el entorno empresarial en pro del avance tecnológico y la competitividad.

\section{Marco conceptual}

Para entender los procesos de facturación electrónica en Colombia es necesario manejar un conjunto de conceptos básicos al cual se hará referencia con frecuencia en el presente documento de análisis. A continuación su definición:

\section{A. Factura electrónica}

Documento electrónico que soporta transacciones de venta de bienes y/o servicios que debe ser expedido, entregado, aceptado y conservado en medios totalmente electrónicos.

\section{B. Obligado a facturar}

Persona natural o jurídica que, debido a las normas tributarias aplicables, se encuentra en la obligación de facturar sus productos y/o servicios asociados a su actividad económica.

\section{Adquiriente}

Persona natural o jurídica que como adquiriente de bienes o servicios deberá exigir una factura electrónica para su rechazo o aceptación y posterior exhibición.

\section{Tercero}

Persona natural o jurídica encargada de prestarle al obligado a facturar los servicios de generación, envío, aceptación, custodia y exhibición de facturas en formato electrónico.

\section{E. Número de la factura electrónica}

Identificador para cada documento electrónico que obedece a un sistema de numeración consecutivo autorizado por la Dirección de Impuestos y Aduanas Nacionales (DIAN).

\section{F. DIAN}

Entidad gubernamental encargada de velar por el cumplimiento de las obligaciones tributarias de las personas naturales y jurídicas en el contexto colombiano.

\section{G. Firma digital}

Representa un valor alfanumérico calculado a partir de un mensaje de datos y un certificado digital y sobre el cual existen algoritmos que permiten verificar la integridad del mensaje, vincular al emisor y, con la ayuda de la ley 527 de 1999, garantizar la no repudiación de contenidos.

\section{Aspectos técnicos}

La idea al momento de diseñar un sistema de facturación electrónica se basa en definir un conjunto de actores en los cuales se puedan distribuir las cargas operacionales y en encontrar los mecanismos más eficientes para poder interactuar con sistemas de información contables 
existentes disminuyendo, de esta manera, los niveles de resistencia en la adopción de esta nueva tecnología. Adicionalmente, se busca cumplir a cabalidad con toda la normatividad vigente y descrita en el apartado introductorio del presente documento de análisis.

En el diseño de facturación electrónica propuesto, se modela conceptualmente un proceso de negocio y un formato de facturación. Adicionalmente se detallan los mecanismos de seguridad adoptados por el sistema de acuerdo al formato de facturación electrónica definido. Posteriormente se detallarán los mecanismos de integración y orquestación de los sistemas de información involucrados.

\section{A. Procesos de negocio}

En los procesos actuales de facturación, la DIAN autoriza a las empresas un rango de facturación tanto para las facturas pre-impresas como para la facturación por computador. Es importante no confundir esta última modalidad de facturación con la electrónica ya que únicamente contempla el uso de un software autorizado por la DIAN para la generación, impresión y gestión de los rangos de facturación con el objetivo de facilitar los procesos contables.

Previa autorización de los rangos de facturación y dada una venta de productos o servicios, una empresa utiliza sus sistemas contables para actualizar inventarios, gestionar órdenes de pedidos, actualizar ingresos, generar reportes de impuestos $y$, por supuesto, generar una factura en formato impreso (con los costos y tiempo que esto demanda). El sistema contable utilizado podrá ser simple o complejo dependiendo del tamaño de la empresa obligada a facturar y los niveles de ventas.
Por un lado, podemos encontrar sistemas de información contables basados en Excel o herramientas de ofimática de libre uso y distribución. Por otro lado, se podrán encontrar sistemas contables basados en ERP's de alta adaptabilidad y complejidad como por ejemplo SAP, PeopleSoft, Oracle E-Business Suite, JD Edwards Enterprise, etc.

Impresa una factura de venta de productos o servicios se deberá enviar vía mensajería tradicional al adquiriente para su correspondiente aceptación o rechazo y posterior conservación. Todo este esquema de facturación se podrá visualizar en la siguiente figura:

Figura 1. Escenario tradicional de facturación

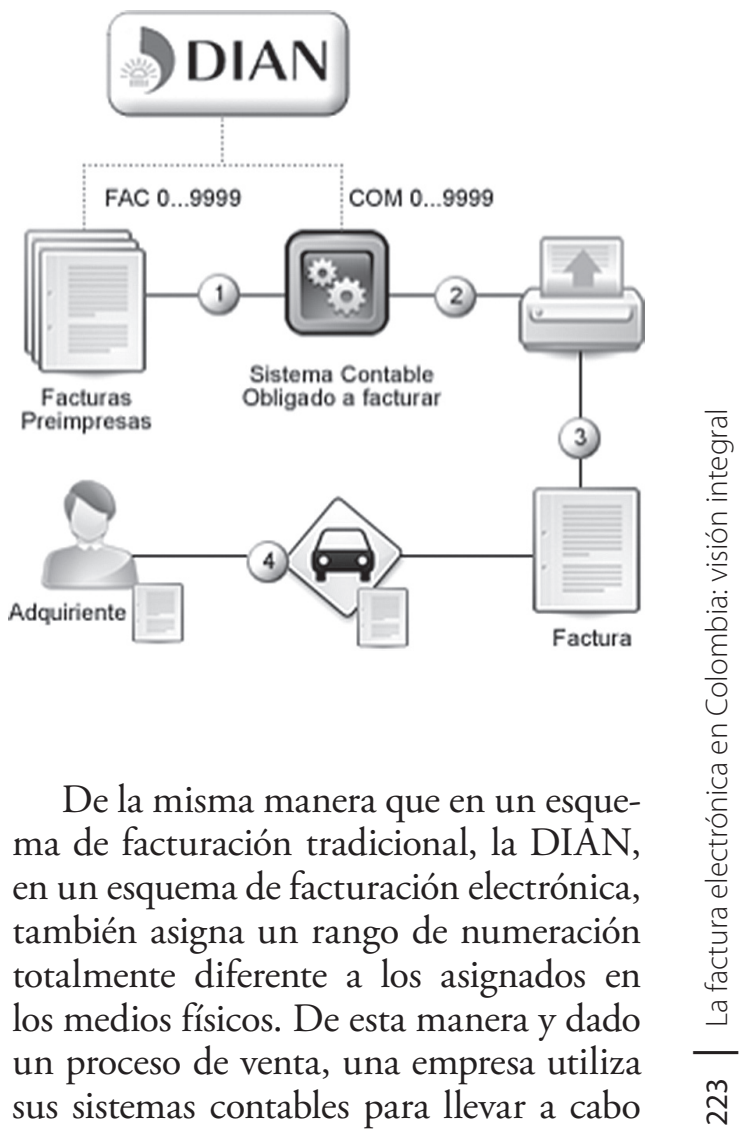


la ejecución de todas las reglas de negocio inherentes a este proceso. Sin embargo, a razón del esquema de facturación electrónica, esta vez no se procederá a generar, imprimir y enviar el documento por medios físicos. En lugar de esto, existirá una orquestación de eventos en los cuales el sistema contable del obligado a facturar notificará a un nuevo sistema de facturación electrónica la necesidad de generar un nuevo documento (la estrategia de comunicación del sistema contable y el sistema de facturación electrónica se abordará en el aparatado Interoperabilidad de este mismo capítulo).

En la notificación de generación de factura, el sistema contable proporcionará los datos mínimos y necesarios para generar un nuevo documento electrónico (la factura). Estos datos incluyen, entre otros, la información del adquiriente, la descripción de los productos adquiridos, los valores de los productos adquiridos $\mathrm{y}$, sobre todo, los impuestos asociados al proceso de venta. Con esta información, el nuevo sistema de facturación electrónica procederá a almacenar la información de facturación en su base de datos (para la generación de los informes bimensuales y detalles que exige la DIAN) y procederá a generar la factura electrónica en dos formatos: el primero para máquinas y sistemas de información y el segundo, para humanos, derivado del primer formato. La factura electrónica en formato "humano" se enviará vía correo electrónico al adquiriente para su aceptación o rechazo y posterior conservación. Adicionalmente, el adquiriente también podrá ingresar al sistema de facturación electrónica para recuperar las facturas que se le han emitido y opcionalmente para generar los reportes de recepción de facturación electrónica y hacer vales, costos y deducciones ante la DIAN. Todo este esquema de facturación se podrá visualizar en la siguiente figura.

Figura 2. Escenario tradicional electrónico.

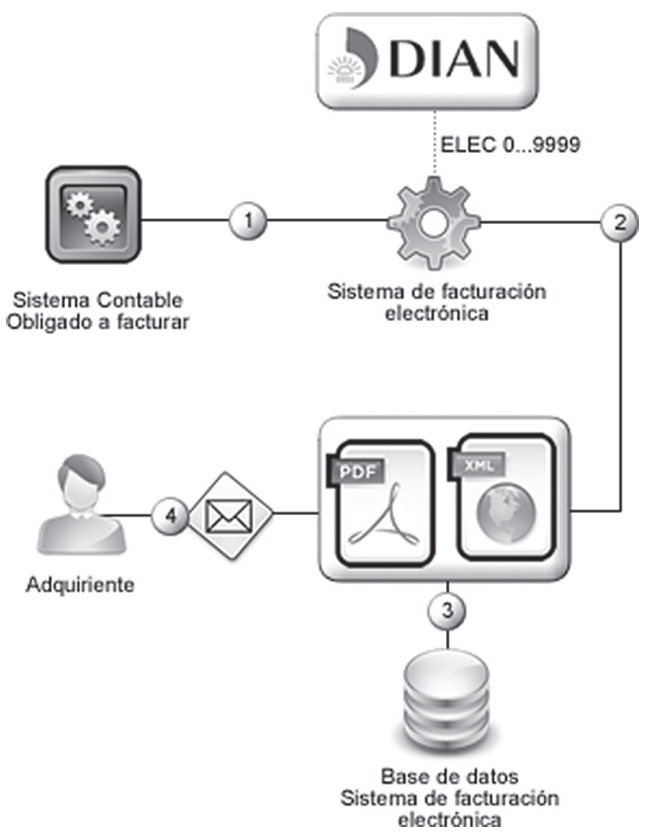

\section{B. Actores involucrados}

Aunque ya se han podido dilucidar tres tipos de actores involucrados en el proceso de facturación electrónica (adquiriente, obligado a facturar y prestador del servicio), un sistema de información de facturación electrónica deberá refinar y distribuir las cargas operativas de este proceso de acuerdo con los patrones generales de software para asignación de responsabilidades en diseño orientado por objetos patrones GRASP (General Responsibility Assignment Software Patterns). Por lo tanto, el sistema de facturación electrónica deberá presentar los siguientes actores: 
- Tercero prestador del servicio: persona encargada de gestionar los obligados a facturar.

- Administrador de la empresa obligada a facturar: persona encargada de gestionar rangos de facturación, certificados digitales y otros actores pertenecientes a la misma empresa (facturadores, auditores contables y auditores funcionales).

- Facturador: persona o sistema de información encargado de enviar las peticiones de facturación electrónica.

- Auditor contable: persona encargada de generar los reportes de facturación a la DIAN (Resolución 14465 - Art. 7 - Anexos técnicos 3, 4 y 5) asociados a la empresa a la cual pertenece.

- Auditor funcional: persona encarga de consultar los logs del sistema y de recuperar y validar la evidencia digital de las facturas asociadas a la empresa a la cual pertenece.

- Adquiriente: persona natural o jurídica receptora de una factura electrónica y que estará encargada de generar de manera opcional sus reportes a las DIAN (Resolución 14465 - Art. 7 - Anexos técnicos 2 y 5) y de consultar las facturas que los obligados a facturar registrados en el sistema le han emitido.

\section{Formato de factura electrónica}

Tal como se mencionó en el parágrafo A (Procesos de negocio) del presente capítulo, un sistema de facturación electrónica deberá estar en la capacidad de generar sus documentos en dos formatos: para máquinas y sistemas de información y para humanos.

La legislación colombiana, a diferencia de la legislación chilena o mexicana, no definió un formato específico que se deberá cumplir para los documentos tributarios como las facturas y notas crédito. Aunque esto representa una ventaja al momento de diseñar un sistema de facturación, también impacta fuertemente la interoperabilidad del sistema con otros sistemas de información, incluidos otros sistemas de facturación.

Por lo tanto, el formato para máquinas se podría definir utilizando un estándar bien conocido como EDIFACT, archivos de textos bien formados de acuerdo a unas reglas de sintaxis preestablecidas o lenguaje XML (muy hablado y entendido por la mayoría de los sistemas de información). Para nuestro caso nos hemos decidido por el formato XML. Sin embargo, existen un millón de maneras de representar una factura con este tipo de formato. A continuación, una buena propuesta.

Una factura electrónica deberá presentar un contenido técnico de control calculado a partir de las directrices dadas en el Anexo No. 1 de la resolución 14465. Adicionalmente, deberá estar asociada a un emisor y un adquiriente, deberá presentar un detalle (productos y servicios facturados) y conjunto de propiedades adicionales de facturación y una buena firma digital.

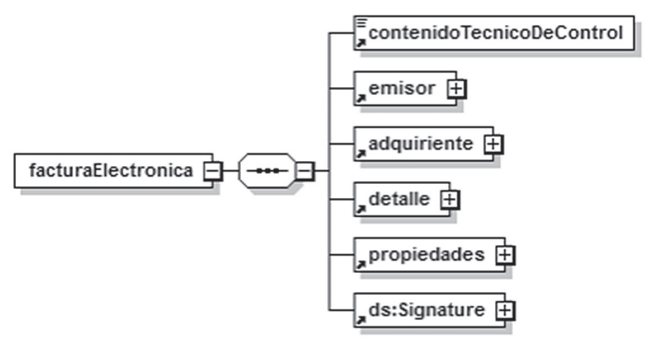


Un emisor de facturación electrónica deberá especificar el nombre o la razón social del tercero que le presta sus servicios de facturación. Adicionalmente, al emisor se le deberá caracterizar con la siguiente información: razón social, NIT, dirección, teléfono, email, sitio web, ciudad y departamento.

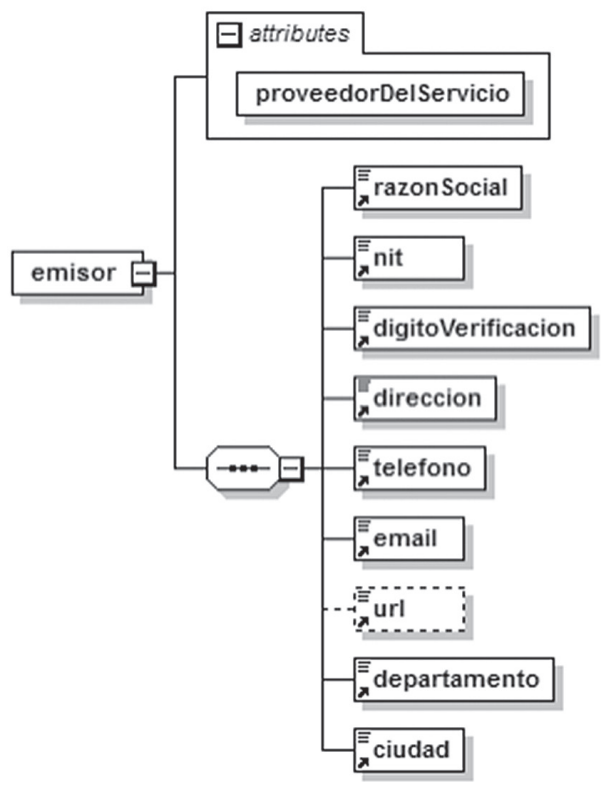

Un adquiriente, por su parte, puede ser de dos tipos: persona natural o persona jurídica. Dependiendo del tipo de adquiriente, la información que caracteriza la entidad varía considerablemente. Por ejemplo, una persona natural presenta una cédula de ciudadanía mientras que una persona jurídica presenta un NIT.

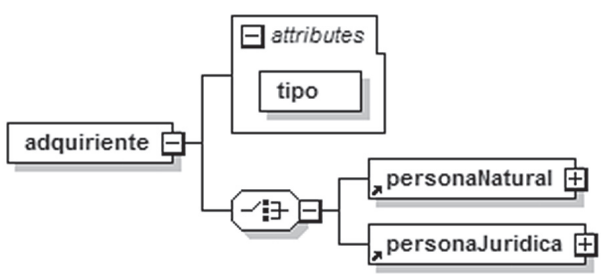

El detalle de una factura electrónica incluye un número de factura que responde a un consecutivo tomado de un rango de facturación debidamente autorizado por la DIAN. Este rango de facturación se define mediante un prefijo, un comienzo de numeración y un final de numeración. De la misma manera y para efectos de conservación y archivo, la factura electrónica deberá presentar una fecha de emisión. Finalmente y con objetivos contables de control y visualización, la factura deberá contener en su detalle el valor total de la transacción, el valor total antes de impuestos, el valor total del IVA (valor sobre el cual la DIAN se encuentra muy interesada), el valor total de otros impuestos (valor sobre el cual la DIAN no se encuentra interesada) y la especificación de los productos o servicios asociados a la transacción. 


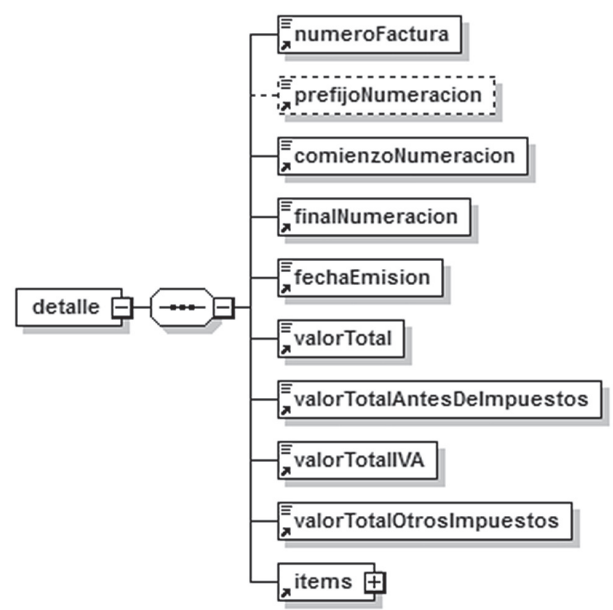

En las propiedades adicionales de una factura se podrá especificar información relevante para la entidad obligada a facturar. Estas propiedades adicionales responden a un punto de extensión necesario en cualquier estándar de facturación. Por ejemplo, por medio de las propiedades adicionales se podrá especificar el nombre del vendedor, el fax del cliente, el número de cuenta para pago por medio de servicios electrónicos, el número de celular asociado a la cuenta, etc.

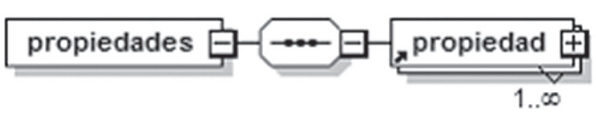

Finalmente, hace falta una entidad de aseguramiento de la información almacenada en el archivo XML. Para nuestro caso se utiliza un estándar de firma digital conocido como XML Signature Enveloped en el cual la firma digital hace parte integral del mensaje de datos.

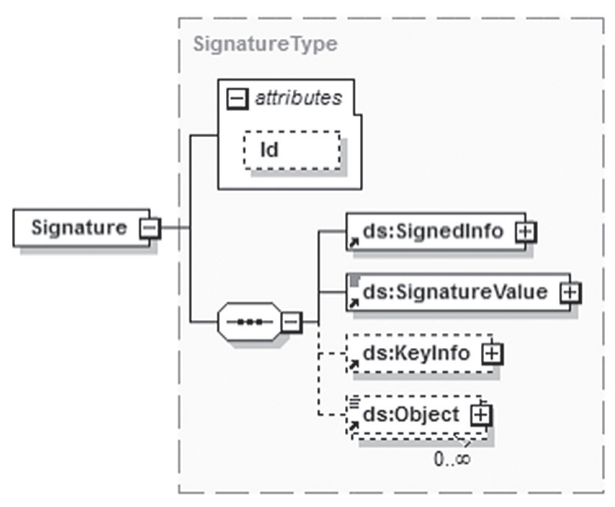

Por otro lado, con respecto al formato para humanos, también se dispone de varias posibilidades: archivos de texto visualmente bien organizados, imágenes, documentos en formato PDF, etc. Para nuestro caso nos hemos decidido por documentos en formato PDF creados a partir del documento XML descrito en los párrafos anteriores. Un documento en formato PDF se puede leer en la mayoría de las plataformas computacionales y sobre todo presenta un soporte nativo de firma digital. Por estas razones es recomendable manejar este tipo de documento electrónico.

\section{Interoperabilidad}

La interoperabilidad de un sistema de facturación electrónica se deberá enfocar desde dos puntos de vista: la integración con los sistemas contables y procesos de negocio de una empresa obligada a facturar y la integración con otros sistemas de facturación electrónica.

\section{Integración con sistemas contables}

Para el primer punto es importante no partir del hecho de que por adoptar nuevas tecnologías es necesario cambiar 
los sistemas de información y procesos existentes. Este es un argumento de venta desenfocado. En lugar de esto, existen formas amigables de negociar la entrada de un nuevo sistema de información el cual representará beneficios en tiempos y costos de facturación.

Para sistemas de información contables, robustos y modernos se puede considerar una arquitectura orientada por servicios (SOA) en la cual el sistema de facturación electrónica publica un Web Service que será consumido por el sistema contable cada vez que se requiera la generación de una factura.

Por otro lado, para sistemas de información Legacy para los cuales el concepto de Web Service no se ha implementado, se podrá considerar una interacción por medio de archivos de texto procesados en Batch. El servicio de procesamiento de estos archivos es parte integral de la solución de facturación electrónica y se basa en un común acuerdo por cuanto la estructura de los archivos de texto armados por el sistema contable.

\section{Integración con otros sistemas de facturación electrónica}

Este es un punto de discusión entre los diferentes proveedores de soluciones de facturación electrónica, ya que la diversidad es tan amplia como proveedores existentes.

Para el autor del presente artículo, el formato XML de facturación electrónica, presentado en el parágrafo anterior, es altamente extensible y consecuente con las necesidades de facturación impuestas por el entorno empresarial y las directivas legales dadas por la DIAN y el Ministerio de Hacienda y Crédito
Público. Sin embargo, para otros proveedores de soluciones, sus formatos de facturación pueden presentar ventajas que no se tuvieron en cuenta en el presente documento de análisis.

Es muy difícil llegar a un acuerdo sin que el Estado colombiano imponga las directivas de construcción del formato de facturación electrónica y notas crédito.

Sin embargo, y de forma independiente a esta situación, el formato de facturación electrónica debe como mínimo presentar tres características fundamentales:

- Información básica de facturación: relacionada con el adquiriente, el obligado a facturar, el valor total de la factura y el valor total de los impuestos.

- Extensibilidad: las facturas varían considerablemente de empresa a empresa. Por lo tanto se deberán proveer los mecanismos para soportar información específica para una empresa dada.

- Firma digital: para dar trámite a las directivas de ley y garantizar la integridad, autenticidad y no repudiación de contenidos.

\section{E. Escenarios de facturación}

Afortunadamente la legislación descrita en la resolución 14465 de 2007 permite la generación de facturación electrónica tanto en entornos netamente empresariales (B2B), en donde el adquiriente es una persona jurídica, como en entornos comerciales (B2C), en donde el adquiriente está representado por una persona natural.

Dada esta característica, un sistema de facturación electrónica deberá soportar de manera adecuada un conjunto de casos de uso que les permitan a 
los dos tipos de actores (empresa y persona natural) llevar a cabo las siguientes operaciones:

- Recuperar las facturas electrónicas que se les han emitido por los diferentes obligados a facturar.

- Aceptar o rechazar las facturas electrónicas.

- Generar los informes correspondientes al anexo No. 3 de la resolución 14465 del 2007.

\section{F. Tecnología PKI aplicada}

De acuerdo al decreto 1929 emitido por el Ministerio de Hacienda y Crédito Público y en particular a su artículo No. 2 en el cual se hace referencia a los principios básicos de autenticidad e integridad, se obliga a todo sistema de facturación electrónica a incorporar mecanismos de firma digital en los documentos tributarios electrónicos como facturas y notas crédito.

Los mecanismos de firma se encuentran fuertemente asociados al formato adoptado para la representación de una factura electrónica. En particular, si se ha adoptado un formato de texto, nada mejor que una firma en formato CMS o PKCS\#7 attached o detached. Eso sí, se deberán también proveer los mecanismos de verificación y extracción del texto claro. Por otro lado, tratándose de documentos en formato XML y PDF no es lo más óptimo o eficiente utilizar los estándares anteriormente referenciados. Los formatos de archivos XML y PDF presentan sus estándares de firma nativos que facilitan los procesos de verificación y visualización de la información firmada digitalmente.
Por un lado, existe el estándar XML Signature el cual viene en tres modalidades: Enveloped, Enveloping y Detached.

En el estándar XML Signature Enveloped la firma digital está contenida dentro de los datos que han sido firmados.

\section{$<$ DocumentoXML> $<$ Signature $>$ $</$ Signature $>$ $</$ DocumentoXML $>$}

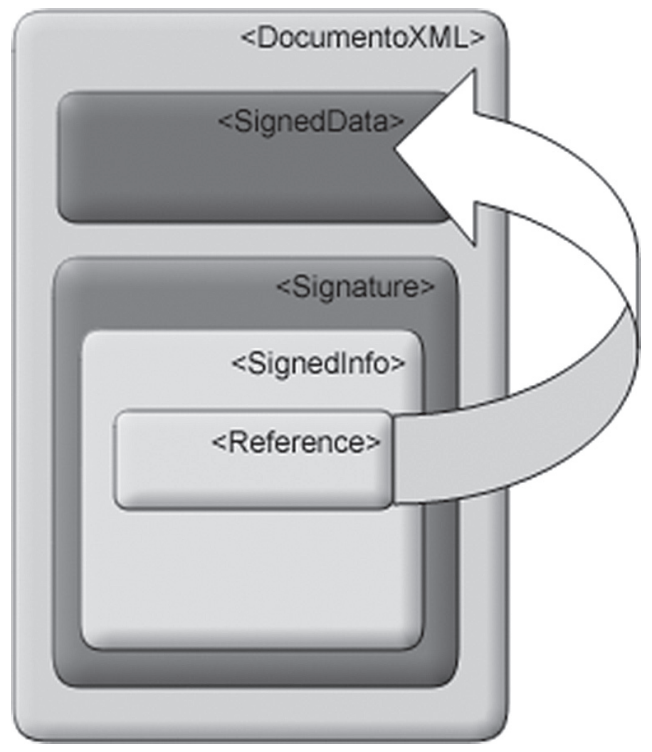

Para el estándar XML Signature Enveloping el documento firmado se adjunta como un nodo adicional del elemento Signature.

$<$ Signature $>$
$\quad<$ DocumentoXML>
$</$ DocumentoXML >
</Signature $>$

$<$ DocumentoXML $>$ $</$ Signature $>$ 


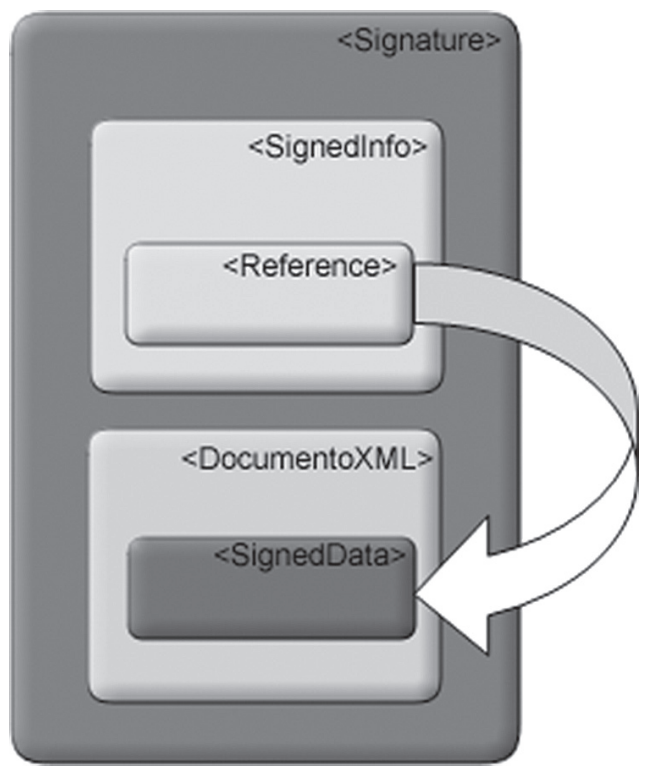

Y, finalmente, para el estándar XML Signature Detached, el elemento firmado es externo a la unidad de firma digital.

\section{$<$ Signature $>$}

$</$ Signature $>$

$<$ DocumentoXML $>$

$</$ DocumentoXML>

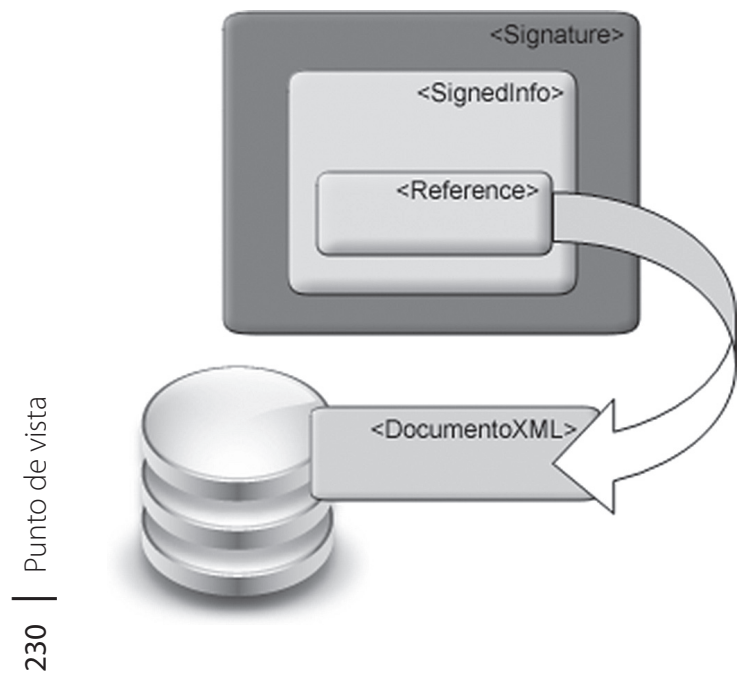

Por otro lado, el formato de archivo PDF maneja de forma nativa los mecanismos integración de firma digital, visualización y verificación. Si bien esta firma digital responde al estándar PKCS\#7, los lectores de documentos PDF saben internamente cómo verificar y visualizar estas firmas sin necesidad de recurrir a software adicional especializado.

\section{Desaciertos}

Existen cuatro puntos que se podrían reevaluar o mejorar en la legislación actual de tal manera que se pueda facilitar la implementación de soluciones de facturación, impulsar el uso de la tecnología en pro de la competitividad industrial a nivel de región y favorecer la interoperabilidad de diferentes soluciones. Estos puntos, en orden de importancia, son: (i) formato de la factura electrónica, (ii) procedimiento de aceptación de facturación electrónica, (iii) proceso de certificación y (iv) contenido técnico de control.

\section{A. Heterogeneidad de formatos de representación de factura electrónica}

Tal como se mencionó anteriormente, la libertad del proveedor en la selección del formato con el cual se representa un documento electrónico tipo factura y nota crédito trae consecuencias negativas en la interoperabilidad de soluciones de facturación. Adicionalmente, los procesos de auditoría y control tomarán un tiempo considerable a razón del desconocimiento del formato a validar y la amplia gama de formatos presente en el mercado. Por estas razones, tanto el Gobierno chileno 
como el mexicano han decido regular e imponer un formato que todos los proveedores interesados en prestar los servicios de facturación electrónica deberán seguir a cabalidad.

\section{B. Aceptación de la factura electrónica}

En México es obligatorio el uso de la factura electrónica para transacciones de venta de más de 2.000 pesos mexicanos. En Colombia, el uso de la facturación electrónica es opcional dependiendo si el adquiriente "acepta" su facturación en medios electrónicos.

El avance empresarial de la región, por medio de la incorporación de nuevas tecnologías, no podrá depender de una decisión tomada por parte del adquiriente. Si éste es una persona natural o jurídica únicamente deberá contar con una conexión a Internet para consultar, aceptar o rechazar sus facturas. Ahora, en lugar de almacenar un documento tributario en formato físico también podrá adaptar medios electrónicos para dar organización e indexación a sus nuevas facturas (con los ahorros de costos y espacio que esto le representa).

Se deberá entender, de entrada, que los cambios siempre presentarán resistencia, sobre todo la incorporación de nueva tecnología en formatos intangibles. Es aquí donde el Estado deberá impulsar (con la obligatoriedad) el uso de estos nuevos medios.

\section{ISO 9001:2000}

En el artículo No. 2 de la resolución 14465 se establece claramente que uno de los requisitos para iniciar operaciones de facturación electrónica es la certificación de calidad ISO 9001:2000 en los procesos de expedición (generación y numeración), entrega, aceptación y conservación, incluyendo la exhibición de la facturación electrónica.

Esta certificación resulta en muchos casos inoperante y se podrá entender como una traba que retrasa considerablemente la entrada y adopción de esta nueva tecnología. En lugar de certificar los procesos, se puede considerar una verificación de la funcionalidad y los casos de uso base del software de facturación electrónica. Los procesos, en muchos casos, pueden estar soportados de forma manual con procedimientos que se encuentran definidos sobre el papel y no se encuentran soportados por software alguno.

\section{Contenido técnico de control}

Aunque el algoritmo descrito en el anexo No. 1 de la resolución 14465 permite verificar técnicamente la integridad de la información contenida en una factura electrónica, también resulta un mecanismo redundante ya que de acuerdo a las disposiciones legales aplicables para este tipo de documentos electrónicos, se debe cumplir con las directivas establecidas en el artículo No. 2 del decreto 1929 que dice: Principios básicos de autenticidad e integridad. La factura electrónica cumple con los principios básicos de autenticidad e integridad, si satisface lo dispuesto en los artículos 8, 9, 16 y 17 de la ley 527 de 1999, en concordancia con los artículos 12 y 13 de la misma ley. Esta información se puede traducir de la siguiente manera: una factura electrónica debe venir acompañada de una firma digital que permita garantizar su autenticidad, integridad y no repudiación en sus 
procesos de emisión, envío, aceptación y conservación.

\section{Conclusiones}

La legislación colombiana da al sector industrial una buena oportunidad de adopción de nuevas tecnologías con la definición de la facturación electrónica. Esta vez no existen vacíos técnicos ni jurídicos ni procedimentales que retrasen la implementación de soluciones que responden a las necesidades empresariales y a las directivas legales.

Sin embargo, se deberán manejar con mucho ingenio y cuidado los mecanismos de interoperabilidad tanto con sistemas de información contables existentes dentro de la organización, como con otros sistemas de facturación electrónica, tarea en la cual el Estado deberá imponer un estándar que todos los proveedores deberán respetar. De la misma manera, la gestión de la evidencia soportada por una firma digital deberá estar debidamente implementada de acuerdo a los formatos de facturación electrónica definidos.

De esta manera y de acuerdo a estas recomendaciones, la factura electrónica impactará positivamente en la gestión de cartera, costos de facturación y en la optimización de procesos logísticos y contables.

\section{Referencias}

I. Adobe, Digital Signatures (2009). Consultado el 2010-10-10, de http://www. adobe.com/security/digsig.html.

2. Adobe, A Prime on Electronic Document Security (2007). Consultado el 2010-06-12, de http://www. adobe.com/security/pdfs/acrobat_livecycle_security_wp.pdf

3. Anexo 20 de la Resolución Miscelánea Fiscal, Legislación Mexicana. Consultado el 2010-06-14, de http:// www.dof.gob.mx/nota_detalle.php?co digo $=5146699 \&$ fecha $=15 / 06 / 2010$

4. Centro de estudios de la economía chilena. Perspectivas de la factura electrónica en Chile. Consultado el 2010-12-01, de http://www.sii.cl/factura_electronica/camara_comercio.pdf

5. Colombia. DIAN. Resolución 14465 (2007). Consultado el 2010-12-04, de http://www.actualicese.com/normatividad/2007/11/28/resolucion-14465de-28-11-2007/

6. Facturación MX, ¿Qué es la factura electrónica? Fundamento Legal. Consultado el 2010-12-04, de http:// www.sat-facturacionelectronica.com. $\mathrm{mx} /$ quees.html

7. Grand, M. Preview of Patterns in Java Volume 2. Consultado el 201012-04, de http://www.mindspring.com / mgrand/pattern_synopses 2 .htm

8. Java Web Services Team, Java XML Digital Signatures (2006). Consultado el 2010-12-05, de http://java. sun.com/developer/technicalArticles/ $\mathrm{xml} /$ dig_signatures/

9. Larman, Grasp patterns. Consultado el 2010-12-06, de http://www. icaen.uiowa.edu/ kuhl/SoftEng/Slides10.pdf 
ro.Ley 527 de 1999. Legislación de comercio electrónico. Consultado el 2010-12-09, de http://www.cej.org. co/component/docman/doc_view/340ley-527-de-1999

I r.Ministerio de Hacienda y Crédito Público Decreto 1929 de 29-052007. Legislación colombiana de facturación electrónica. Consultado el 2010-12-09, de http://www.actualicese.com/normatividad/2007/05/29/decreto-1929-de-29-05-2007/

I2.Nieto, L. Estructura de segmentos EDIFACT y reglas de sintaxis (2010). Consultado el 2010-12-10, de http://www.re-orientation.com/segmentos-estructura-intercambio-edifact

I3. Odete España, Mensajes de EDIFACT (2003). Consultado el 201012-12, de http://www.odette.es/SGC/ downloads/cursoEDI/413.pps

I4. Resolución exenta SII N45 del 01 de septiembre del 2003. Normas y procedimientos de operación respecto de los documentos tributarios electrónicos (2003). Consultado el 201010-06, de http://www.sii.cl/documentos/resoluciones/2003/reso $45 . h t m$

I 5. Sun Microsystems, Using the Java XML Digital Signature API. Consultado el 2010-07-13, de http:// www.java-tips.org/java-ee-tips/xml-digital-signature-api/using-the-java-xmldigital-signatur-2.html

I6. W3C, XML Signature Syntax and Processing. Consultado el 2010-1201, de http://www.w3.org/TR/xmldsig-corel

17. W3C, XML Signature. Consultado el 2010-07-06, de http://www.w3.org/ Signature/

I8. Worcester Polytechnic Institute, Grasp patterns. Consultado el 201008-22, de http://www.cs.wpi.edu/_gpo llice/cs4233-a05/CourseNotes/maps/ class4/GRASPpatterns.html 